\title{
Vibration Control of Tall BuILdings Using Mega SubConfiguration
}

\author{
By Maria Q. Feng, ${ }^{1}$ Associate Member, ASCE, and Akira Mita, ${ }^{2}$ Member, ASCE
}

\begin{abstract}
An innovative vibration-control system is proposed to reduce the dynamic response of tall buildings to wind and seismic loads. This system takes advantage of the so-called megasubstructure configuration, which is especially popular in tall buildings. Substructures contained in the megastructure serve as energy absorbers so that no additional mass is required for the intended vibration control as seen in the conventional mass damper systems. The proposed system naturally resolves the difficulties in augmenting damping capacities of tall buildings associated with the high rigidity and deformation in the dominant bending mode. Dynamic characteristics of the proposed control system including the frequency response and the energy flow are investigated. Optimal values of structural parameters such as the damping ratio and stiffness of the substructure are determined. The feasibility and effectiveness of this unique control system in improving human comfort and protecting structures under both wind and earthquake loads are demonstrated through analytical and numerical analysis.
\end{abstract}

\section{INTRODUCTION}

Traditionally, architects and engineers have been interested in constructing structurally sound, functionally efficient, and esthetically elegant high-rise buildings. Remarkable advances made over recent years in engineering science including materials, geotechnical, and structural engineering have benefited the analysis, design, and construction of tall buildings. Such advances, it appears, continue to accelerate to the extent that construction of super tall buildings, as high as $1,000 \mathrm{~m}$ will become possible in the near future if certain technical challenges can be met.

One major issue that constitutes a technical challenge is the problem of ensuring the structural integrity of super tall buildings under seismic and wind loads, because super tall buildings are anticipated to suffer from excessive vibrations under these natural hazards. Such vibrations are primarily due to the combination of slenderness in building configuration and the lack of damping capacity. Obviously, for structural safety, the buildings should be designed to separate their natural frequencies from dominant seismic frequencies as well as from possible wind-induced vibration frequencies, including frequencies resulting from vortex shedding. In addition to meeting the demands of structural safety, however, the building vibration also needs to be contained within a human comfort threshold particularly in a sustained wind environment.

Structural characteristics common to most super tall buildings, such as the high rigidity of the structural system and the dominant bending deformation, result in technical difficulties, which, for example, prevent the application of conventional damping devices. The high rigidity requires a large number of damping devices in order to achieve a certain desirable damping ratio. A major component of the deformation associated with the fundamental mode is the bending deformation so that damping devices utilizing shear deformation would no longer be effective. Even if a damping device effective for bending deformation could be developed, the

\footnotetext{
'Asst. Prof., Dept. of Civ. and Envir. Engrg., Univ. of California, Irvine, CA 92717

'Sr. Res., Izumi Res. Inst., Shimizu Corp., 2-2-2 Uchisaiwai-cho, Chiyoda-ku, Tokyo 100 Japan.

Note. Associate Editor: Mircea D. Grigoriu. Discussion open until March 1, 1996. To extend the closing date one month, a written request must be filed with the ASCE Manager of Journals. The manuscript for this paper was submitted for review and possible publication on August 26. 1994. This paper is part of the Journal of Engineering Mechanics, Vol. 121, No. 10, October, 1995. CASCE ISSN 0733-9399/95/0010-1082$1088 / \$ 2.00+\$ .25$ per page. Paper No. 9125
}

number of damping devices needed would be extraordinarily large and, thus, its use would be economically impractical.

The tuned mass damper system has been applied to many actual buildings in which an additional mass is attached to a building to suppress vibration dynamically. Such systems usually control the fundamental mode only and, hence, they are not necessarily effective for seismically induced vibration. Further, these systems are not safe for use in super tall buildings. As a building gets taller and more massive, a heavier additional mass is required and a larger stroke of this mass is anticipated; this large stroke raises significant safety concerns. The active or hybrid mass damper system may control multiple vibration modes and can relax the requirements for strokes and/or masses. However, its capacity is limited to ensure human comfort but not to protect the building structure itself under strong winds and earthquakes, because of the power limitations of actuators. In addition, the reliability concerns and high cost of active and hybrid systems prevent these systems from being implemented in actual buildings.

In this paper, a new method of vibration control for tall buildings is presented, which takes advantage of the megasubstructural configuration and does not require additional mass or active control devices (Mita and Feng 1994). This method overcomes the difficulties associated with the vibration control of tall and super tall buildings in an innovative manner, as described in the following section.

\section{PROPOSED VIBRATION-CONTROL SYSTEM}

The proposed vibration-control system takes advantage of the mega-substructure configuration of tall buildings. A typical megasubstructure is shown in Fig. 1, in which a conventional rigid frame system is also shown for comparison. The megasubstructure configuration is becoming popular in the profession, especially for the design and construction of super tall buildings because this system exhibits structural efficiency by allowing high rigidity of the structure while minimizing the amount of structural materials to be used.

The megastructure contains substructures that consist of several floors. Taking advantage of this structural configuration, a new response-control strategy is established as follows: First, the vibration energy (kinetic energy) of the megastructure due to wind or seismic loads is transferred into substructres. Second, the transferred energy is dissipated in the substructure. The first step can be achieved by tuning the dynamic characteristics of substructures so that most kinetic energy flows naturally into the substructures. The second step can be easily established by designing the substructures to vibrate in shear modes so that any conventional damping 


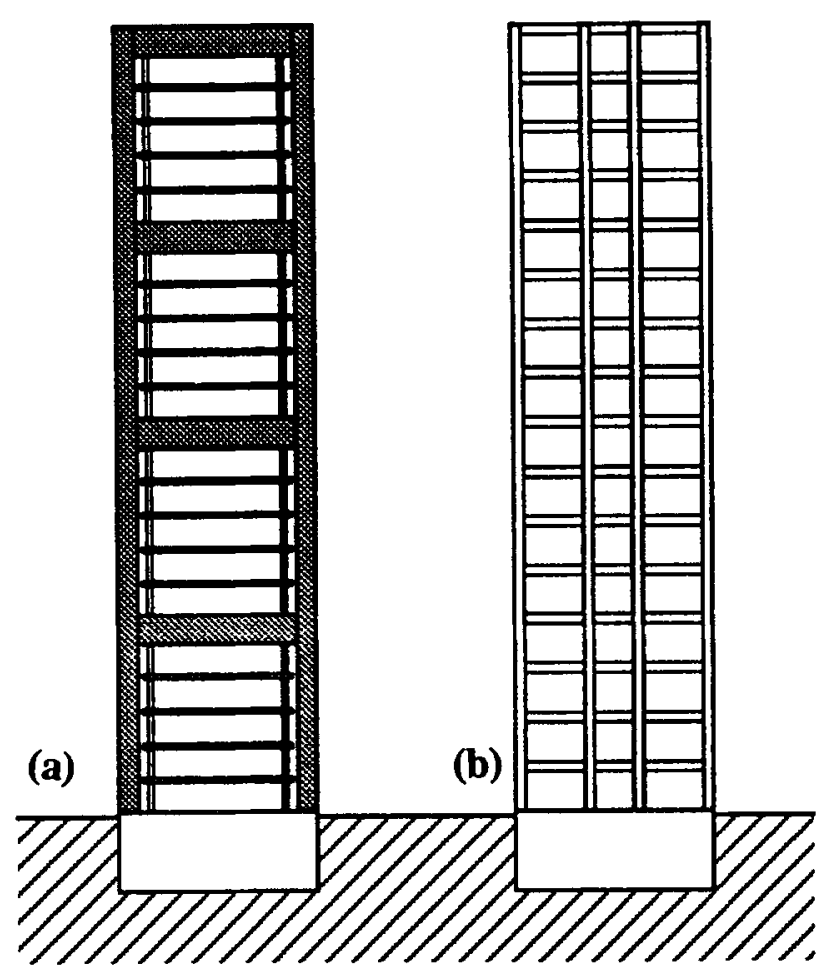

FIG. 1(a). Mega Substructure; (b) Conventional Rigid Frame

devices could be used for energy dissipation in the substructures.

The proposed control system has the following features, which are not observed in the conventional mass damper systems: (1) The substructures themselves serve as absorbers and, thus, the mass ratio achieved by the proposed system can be significantly larger than the mass ratio of the conventional mass damper system, which is typically of the order of one to several percent. The large mass ratio implies that an extremely high level of response reduction in not only the megastructure but also the substructures can be achieved; (2) the substructures naturally have several vibration modes; thus, energy in a broad frequency range can be absorbed by the substructures. This cannot be realized by the conventional mass damper system; (3) the proposed control system does not require any additional mass. To distinguish it from the usual mass damper system, the proposed system is called the mega-subcontrol system.

In the design of the mass damper system, the motion characteristics of the tuned mass itself are no major concern except for the amount of its stroke. In the case of the mega subsystem, however, the vibration of the substructures, which actually corresponds to the tuned mass in the mass damper system, also needs to be controlled to a low level to improve the comfort of occupants and to protect vibration-sensitive equipment and other nonstructural components housed in the substructures. Therefore, the control objective here is dual not only to suppress the vibration of the megastructure for structural safety purpose but also to minimize the vibration of the substructures. For this reason, it is essential that extra care be exercised in designing the proposed mega-subcontrol system.

\section{DYNAMIC CHARACTERISTICS}

To demonstrate the effectiveness of the proposed control method, the dynamic characteristics of the mega subsystem are studied and compared with the mass damper system. For simplicity but without loss of generality, a megastructure and a substructure are modeled by a single-degree-of-freedom

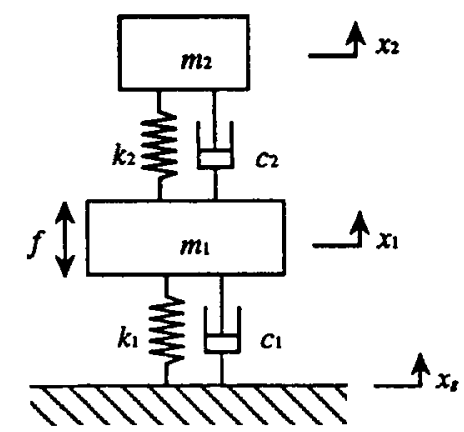

FIG. 2. Analytical Model

system, respectively (as shown in Fig. 2), in which $m_{1}$ represents the mass of the megastructure and $m_{2}$ represents the mass of the substructure. This is the same simplified model as the conventional tuned mass damper system in which $m_{1}$ is the mass of the building and $m_{2}$ is the mass of the mass damper. However, the difference in the value of the mass ratio (one is of the order of $1 \%$ and the other of $100 \%$ ) makes the dynamic characteristics of the mega substructure significantly more advantageous.

\section{Frequency Response}

The equation of motion of the simplified system subject to the wind load $f$ can be written in the form

$$
\begin{gathered}
{\left[\begin{array}{cc}
m_{1} & 0 \\
0 & m_{2}
\end{array}\right]\left\{\begin{array}{l}
\ddot{x}_{1} \\
\ddot{x}_{2}
\end{array}\right\}+\left[\begin{array}{cc}
c_{1}+c_{2} & -c_{2} \\
-c_{2} & c_{2}
\end{array}\right]\left\{\begin{array}{l}
\dot{x}_{1} \\
\dot{x}_{2}
\end{array}\right\}} \\
+\left[\begin{array}{cc}
k_{1}+k_{2} & -k_{2} \\
-k_{2} & k_{2}
\end{array}\right]\left\{\begin{array}{l}
x_{1} \\
x_{2}
\end{array}\right\}=\left\{\begin{array}{l}
f \\
0
\end{array}\right\}
\end{gathered}
$$

where $x_{1}$ and $x_{2}=$ relative displacements of the mega and substructures with respect to the ground. In case of the seismic load, the forcing term of the equation should be modified accordingly. The natural frequencies and the damping ratios of the mega and substructures are defined as follows:

$$
\omega_{1}=\sqrt{\frac{k_{1}}{m_{1}}} ; \quad \omega_{2}=\sqrt{\frac{k_{2}}{m_{2}}} ; \quad h_{1}=\frac{c_{1}}{2 m_{1} \omega_{1}} ; \quad h_{2}=\frac{c_{2}}{2 m_{2} \omega_{1}}
$$

The damping ratio of the substructure $h_{2}$ is defined with reference to $\omega_{1}$ instead of $\omega_{2}$. The mass ratio and the frequency ratio are represented by the following parameters:

$$
\mu=\frac{m_{2}}{m_{1}} ; \quad \beta=\frac{\omega_{2}}{\omega_{1}}
$$

In Fig. 3, the amplitudes of the transfer function between the velocity response of the mega substructure and the excitation force are depicted for different mass ratio $\mu=0.01$, 0.1 , and 1.0. The parameters used for this example are given by

$$
\begin{aligned}
& \omega_{1}=1.0(\mathrm{rad} / \mathrm{s}) ; \quad h_{1}=0.01 \\
& \beta=\sqrt{\frac{1}{1+\mu} ; \quad h_{2}=\beta \frac{\sqrt{\mu}}{2}}
\end{aligned}
$$

where the frequency ratio $\beta$ and the damping ratio of the substructure $h_{2}$ are selected as optimum to minimize the velocity response of the megastructure when the wind force is modeled by white noise (Warburton 1982). Fig. 3(a) gives the velocity transfer function of the megastructure to the excitation force, and Fig. 3(b) gives the velocity transfer function of the substructure. It is clearly indicated that the system 

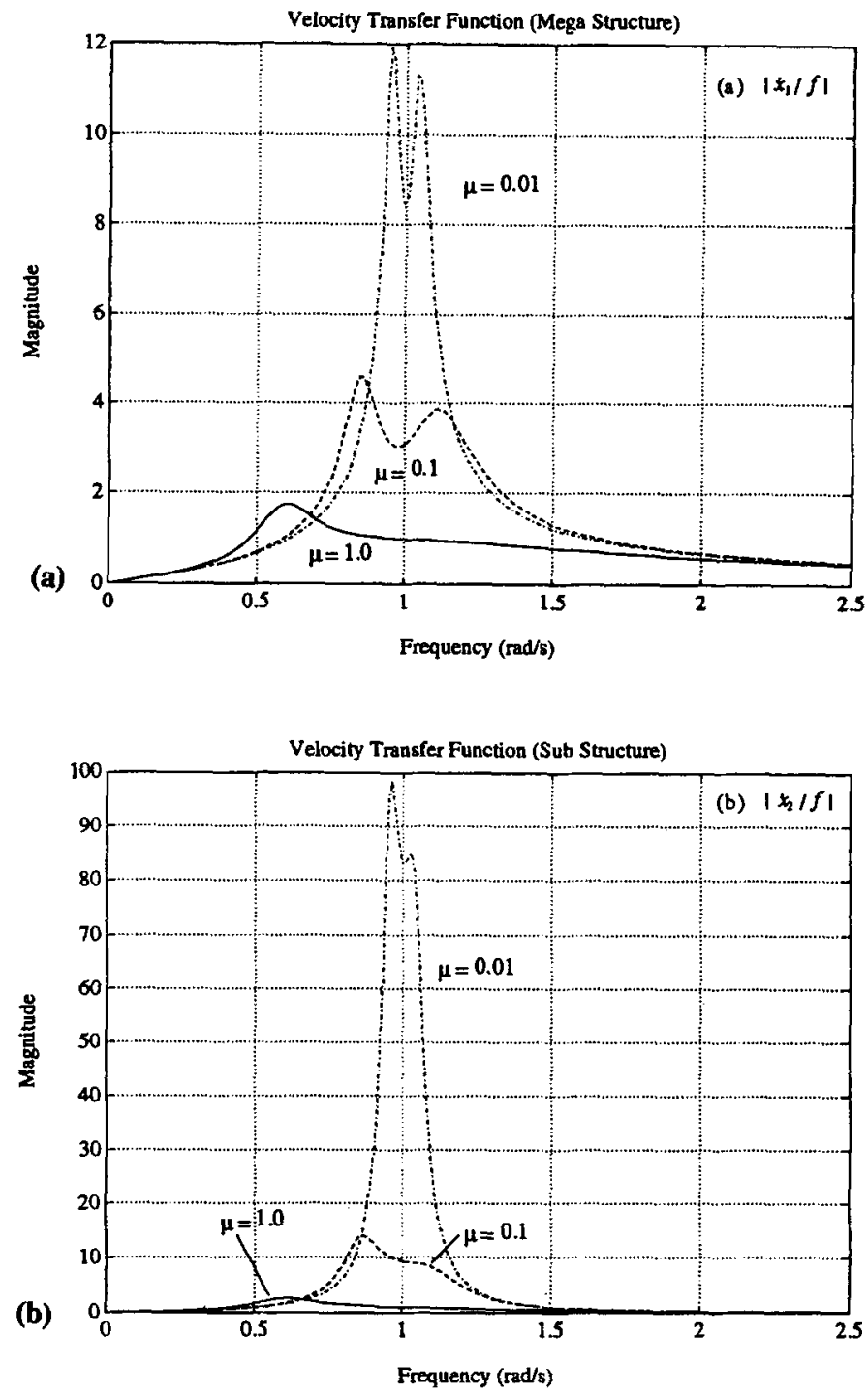

FIG. 3. Transfer Functions

with a large mass ratio makes the velocity response of not only the mega but also the substructure significantly reduced over a much broader frequency range.

\section{Energy Flow Characteristics}

To explain why a large mass ratio can reduce vibration response in both mega and substructures, the energy flow from the megastructure into the substructure is investigated. In Figs. 4(a) and (b), the energy flows for two mass ratios $m=0.01$ (mass damper system) and 1.0 (mega subsystem) are presented. The total energy absorbed by the substructure (or mass damper) is decomposed into two components, one is transferred through the spring and the other through the dashpot. The energy flow shown in Fig. 4 is the energy transferred from the megastructure into the substructure per unit second when the megastructure is vibrating with the unit velocity amplitude. The negative energy means that the energy flows from the substructure into the megastructure, instead of from the megastructure into the substructure. This phenomenon is observed on the dashpot element in the low-frequency range. For details of the definition of energy flow, see Mita and Kaneko (1992) and Kaneko and Mita (1993).

Fig. 4 demonstrates that the amount of energy flow from the megastructure to the substructure is much larger over a much wider frequency range for the system with a large mass ratio, i.e., the mega subsystem. This is the reason for the
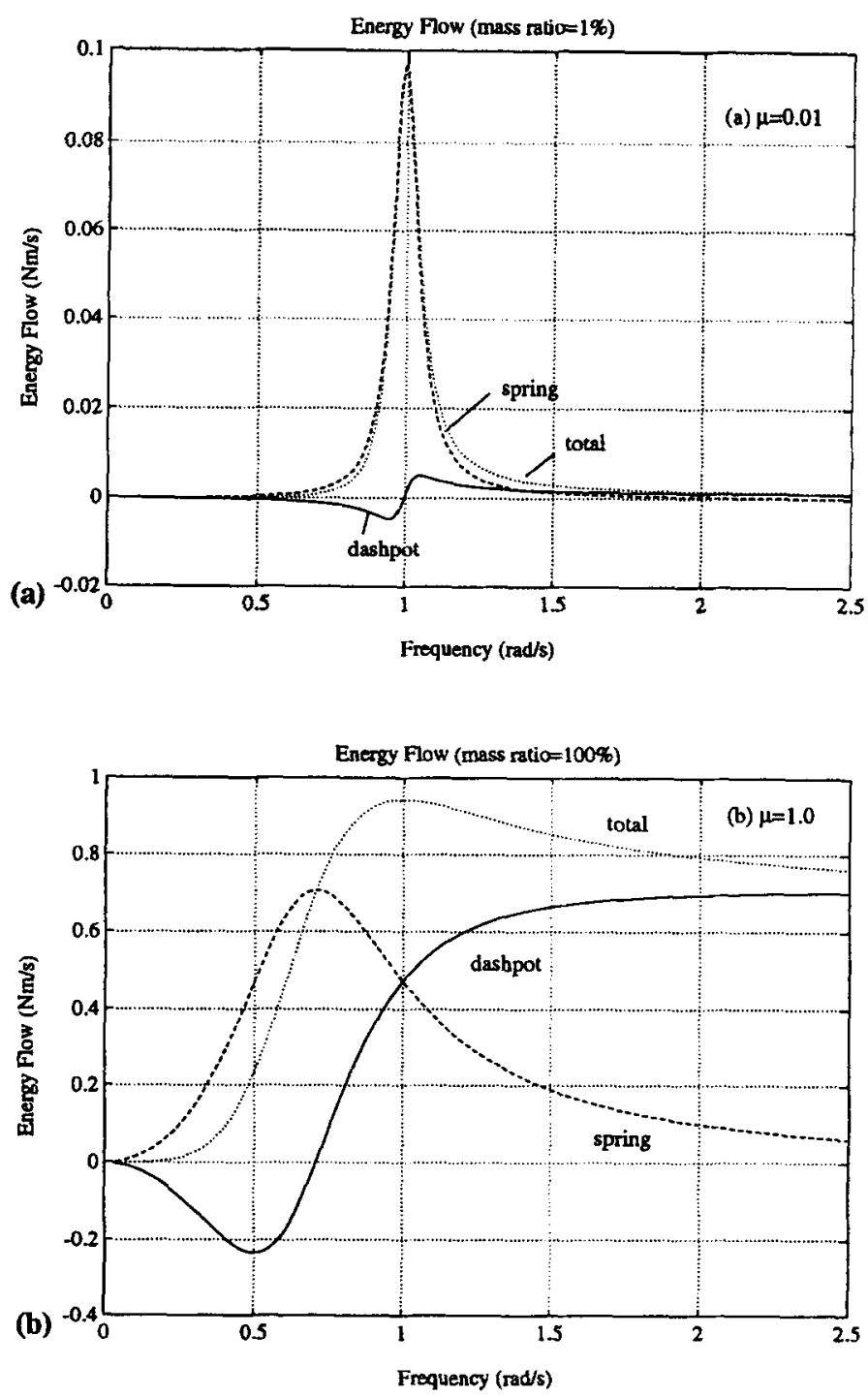

FIG. 4. Energy Flow from Megastructure into Substructure

vibration response of the megastructure (which has a large mass ratio) being much smaller than that of the mass damper system (which has a small mass ratio). More importantly, the detail of the energy flow is different for different mass ratios. In the case of mass ratio 0.01 , most of the energy is transferred through the spring, implying that in the mass damper system the energy is first transferred into the kinetic energy of the damper and then dissipated by the dashpot. As opposed to that, in the case of mass ratio 1.0, most of the energy is directly dissipated through the dashpot before it is transferred to the kinetic energy of the subsubstructure. This is the reason why the vibration response of the substructure can be smaller in the mega subsystem than in the mass damper system.

\section{Robustness}

The square root of the mean square displacement response of the megastructure $\sqrt{E\left[x_{1}^{2}\right]}$ subject to the white-noise wind load of unit spectral intensity is given in Figs. 5(a) and (b) for small $(0.01)$ and large (1.0) mass ratios as a function of the frequency ratio $\left(\beta=\omega_{2} / \omega_{1}\right)$ and damping ratio $\left(h_{2}\right)$. Comments on the white-noise assumption for the wind load will be made later. The square root of the mean square acceleration response of the substructure $\sqrt{E\left[\ddot{x}_{2}^{2}\right]}$ for the same external load is depicted in Fig. 6. These figures demonstrate the robustness of the proposed system, which has a large mass ratio. Fig. 5(a) shows that the parameter range in which the 

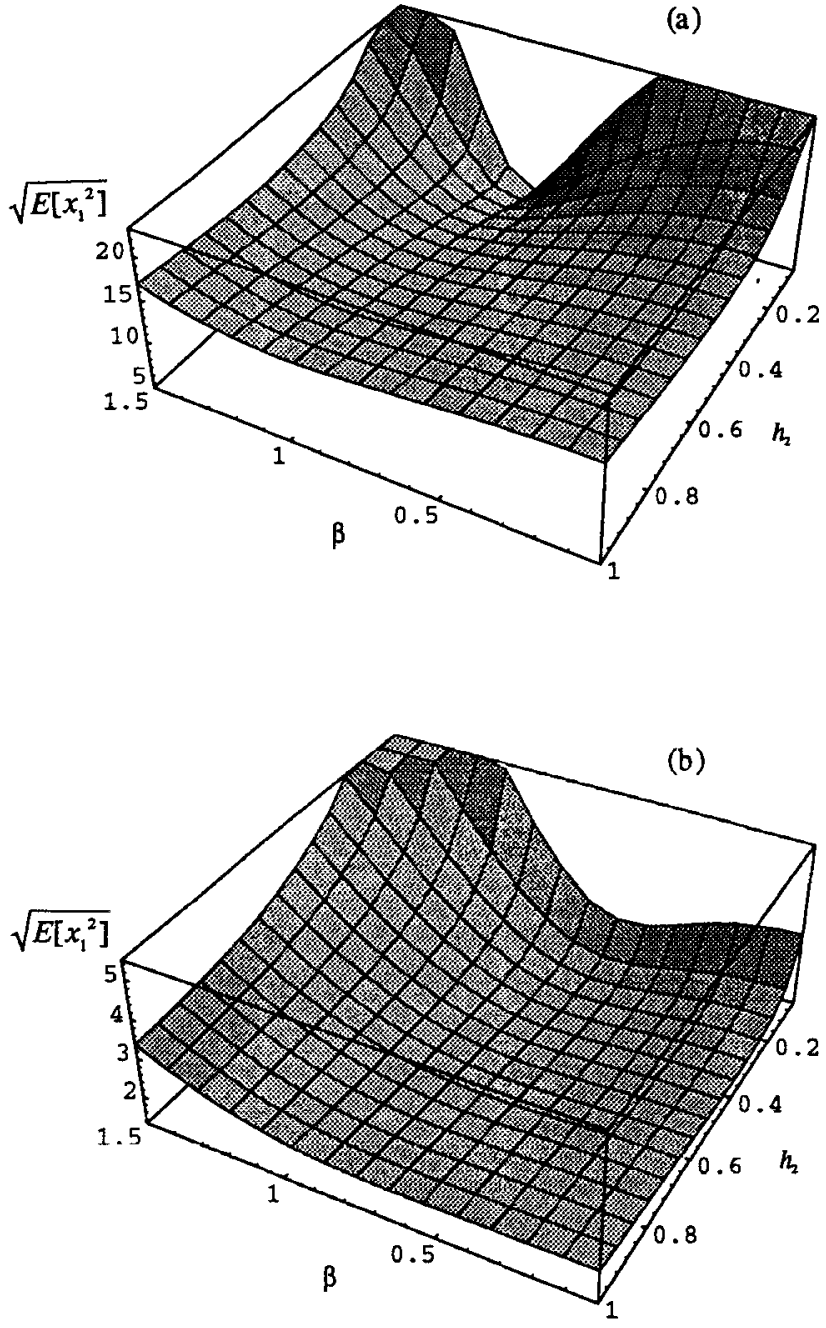

FIG. 5. Response Displacement of Megastructure to Wind Loads: (a) $\mu=0.01$; (b) $\mu=1.0$

system can be effective is very narrow for the small mass ratio. In contrast to that, both Figs. 5(b) and 6(b) indicate that for the system with a large mass ratio, the parameter range in the $\beta-h_{2}$ domain where the response is small is significantly wide. Particularly, when the natural period of the substructure is relatively long and the damping ratio is relatively large, the proposed control system can be quite robust and effective.

\section{OPTIMUM PARAMETERS}

As depicted, the advantages of a larger mass ratio in the mega substructure can be exploited to develop a very effective and robust vibration-control system for tall and super tall buildings. Parameters such as the damping ratio and frequency of the substructure, however, need to be studied to achieve an optimum performance. The optimum parameters can be derived for wind and earthquake loads, depending on control criteria.

\section{Control Criteria}

There are different control criteria depending on different dynamic loads and response quantities of interest. Generally speaking, if the building is subjected to a low to medium wind or a far-field earthquake of long duration, the absolute acceleration of the substructure needs to be reduced to improve the comfort of occupants. However, for a high wind or a near-field earthquake of strong intensity, the priority of the
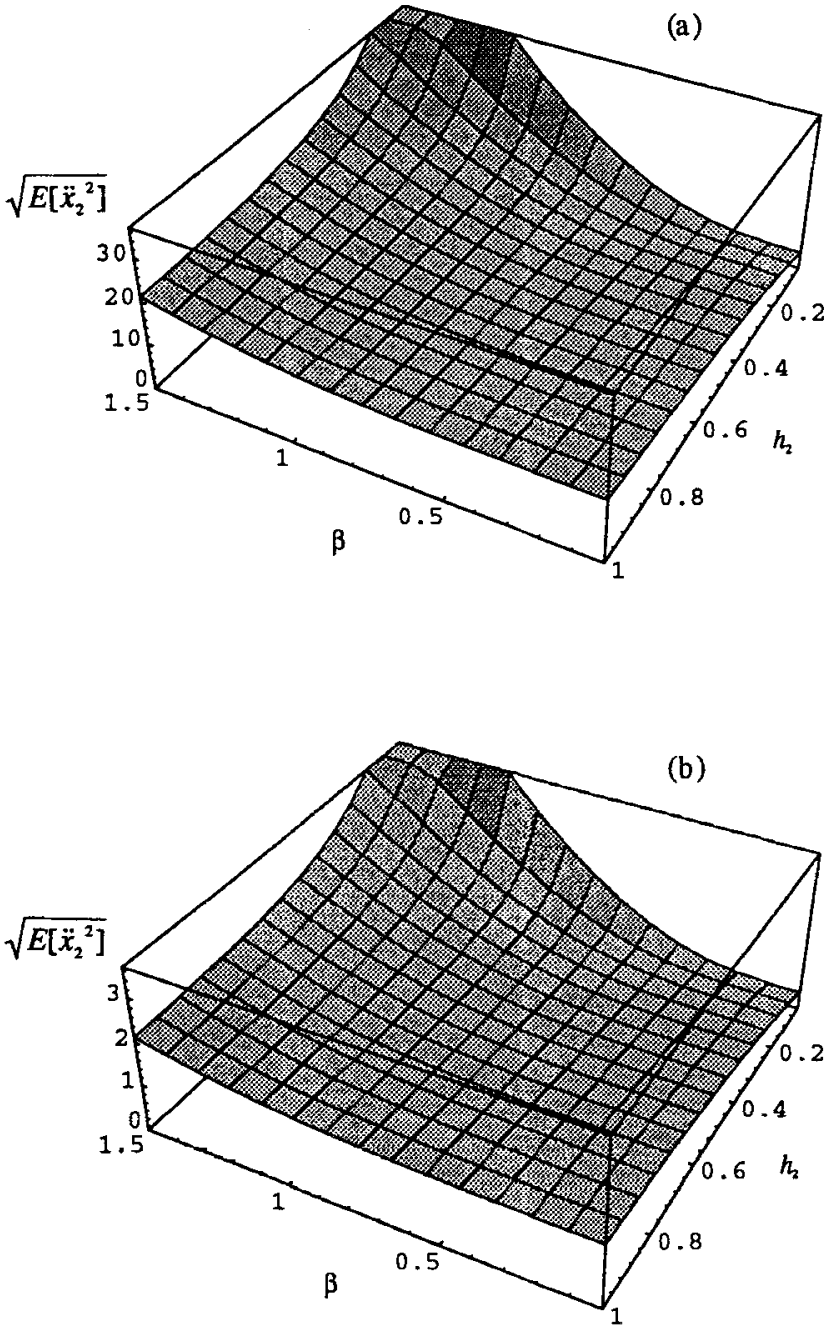

FIG. 6. Response Acceleration of Substructure to Wind Loads: (a) $\mu=0.01$; (b) $\mu=1.0$

control objective changes to the reduction of mega deformation to protect the structure itself. Therefore, in the high wind or near-field earthquake cases, the control objectives are to minimize the subacceleration and megadisplacement.

To obtain the analytical solution for the optimal values of the parameters, which minimize the dynamic response of the building measured in terms of the mean square values of the megadisplacement $E\left[x_{1}^{2}\right]$ and the subacceleration $E\left[\ddot{x}_{2}^{2}\right]$, it is assumed that the earthquake ground motion and the fluctuating component of the wind-induced pressure force are the white noise and that the megastructure has zero damping. In case of the wind excitation, the white-noise excitation is not an unreasonable assumption, because the tall building vibrates primarily in the first mode under high-wind conditions around its statically deflected position under the static component of the wind pressure.

\section{Optimization}

For the two-degree-of-freedom system shown in Fig. 2, the mean square $E\left[x^{2}\right]$ of the response $x$, when subjected to the white-noise excitation of the power spectral density $S_{0}$, is known as (Crandall and Mark 1963)

$$
E\left[x^{2}\right]=S_{0} \int_{-\infty}^{\infty}\left|H_{x}(\omega)\right|^{2} d \omega
$$

where $H_{x}(\omega)=$ complex transfer function in the form 


$$
H_{x}(\omega)=\frac{-i \omega^{3} B_{3}-\omega^{2} B_{2}+i \omega B_{1}+B_{0}}{\omega^{4} A_{4}-i \omega^{3} A_{3}-\omega^{2} A_{2}+i \omega A_{1}+A_{0}}
$$

For different sources of excitation and different transfer functions, the coefficients $B_{0}, B_{1}, B_{2}$, and $B_{3}$ are different as given in Table 1 , while the coefficients $A_{0}, A_{1}, A_{2}, A_{3}$, and $A_{4}$ have the same values as the following:

$$
\begin{gathered}
A_{0}=\beta^{2} \omega_{1}^{4} ; \quad A_{1}=2 h_{2} \omega_{1}^{3} ; \quad A_{2}=\left[(1+\mu) \beta^{2}+1\right] \omega_{1}^{2} \\
A_{3}=2(1+\mu) h_{2} \omega_{1} ; \quad A_{4}=1
\end{gathered}
$$

The integration in (5) leads to

$$
\begin{aligned}
& E\left[x^{2}\right]=S_{00} \pi\left\{\left[\left(B_{01}^{2} / A_{0}\right)\left(A_{2} A_{3}-A_{1} A_{4}\right)+A_{3}\left(B_{1}^{2}-2 B_{0} B_{3}\right)\right.\right. \\
& \quad+A_{1}\left(B_{2}^{2}-2 B_{1} B_{3}\right)+\left(B_{3}^{2} / A_{4}\right)\left(A_{1} A_{2}\right. \\
& \left.\left.\left.\left.\quad-A_{0} A_{3}\right)\right] / A_{1}\left(A_{2} A_{3}-A_{1} A_{4}\right)-A_{0} A_{3}^{2}\right]\right\}
\end{aligned}
$$

The optimizing conditions are

$$
\frac{\partial E\left[x^{2}\right]}{\partial \beta}=0 ; \quad \text { and } \quad \frac{\partial E\left[x^{2}\right]}{\partial h_{2}}=0
$$

Applying these conditions, simple expressions for the optimal values of the frequency ratio $\beta_{\text {opt }}$ and damping ratio $h_{2 \mathrm{opt}}$ are obtained for different responses to be optimized under different sources of excitation. To decouple the equations for optimization, the damping ratio $h_{1}$ is assumed to be zero. The optimal parameters are given in Table 2 .

In the expression of the optimal damping ratio $h_{2 \mathrm{opv}}$, the optimal frequency ratio $\beta_{\text {opt }}$ is not substituted. The reason $\beta$ is left as it is in the expression $h_{2 o p t}$ is for design convenience. For example, the optimal $\beta_{\text {opt }}$ should be zero in order to minimize the response acceleration of the substructure. However, in practice, zero frequency is impossible to realize and, therefore, in this case, design parameters should be chosen within their practical ranges, yet should produce a most favorable value of frequency. The expression $h_{2 \text { opt }}$, which contains $\beta$ provides convenience for that purpose.

The optimal parameter values listed in Table 2 can be con-

\begin{tabular}{|c|c|c|c|}
\hline $\begin{array}{c}\text { Excita- } \\
\text { tion } \\
\text { (1) }\end{array}$ & $\begin{array}{l}\text { Response } \\
\text { to be } \\
\text { optimized } \\
\text { (2) }\end{array}$ & $\begin{array}{l}\beta_{\text {opt }} \\
\text { (3) }\end{array}$ & $\begin{array}{l}h_{\text {2opt }} \\
\text { (4) }\end{array}$ \\
\hline$f$ & $\begin{array}{l}x_{1} \\
\ddot{x}\end{array}$ & $\frac{\sqrt{1+(\mu / 2)}}{(1+\mu)}$ & $\begin{array}{l}\frac{1}{2} \sqrt{(1+\mu) \beta^{4}-\frac{2+\mu}{1+\mu} \beta^{2}+\frac{1}{1-\mu}} \\
\frac{\beta^{2}}{2}\end{array}$ \\
\hline$\ddot{x}_{g}$ & $\begin{array}{l}x_{1} \\
\ddot{x}_{2}+\ddot{x}_{k}\end{array}$ & $\begin{array}{l}\frac{\sqrt{1-(\mu / 2)}}{(1+\mu)} \\
0\end{array}$ & $\left\{\begin{array}{l}\frac{1}{2} \sqrt{(1+\mu) \beta^{4}+\beta^{2}+\frac{1-3(1+\mu)^{2} \beta^{2}}{(1+\mu)^{3}}} \\
\frac{\beta}{2} \sqrt{(1+\mu) \beta^{2}+1-\frac{1}{1+\mu}}\end{array}\right.$ \\
\hline
\end{tabular}
firmed by the plots $\sqrt{E\left[x_{1}^{2}\right]}$ and $\sqrt{E\left[\ddot{x}_{2}^{2}\right]}$ shown in Figs. 5 and 6.

The optimization study in the current stage is based on the

TABLE 1. Coefficients in Transfer Function

\begin{tabular}{c|l|l|l|l|l}
\hline \hline $\begin{array}{c}\text { Excita- } \\
\text { tion } \\
(1)\end{array}$ & $\begin{array}{c}\text { Transfer } \\
\text { functions } \\
(2)\end{array}$ & \multicolumn{1}{|c|}{$B_{0}$} & \multicolumn{1}{|c|}{$B_{1}$} & \multicolumn{1}{c|}{$B_{2}$} & \multicolumn{1}{c}{$B_{3}$} \\
& \multicolumn{1}{c|}{$(3)$} & \multicolumn{1}{c}{$(4)$} & $(5)$ & $(6)$ \\
\hline$f$ & $x_{1} / f$ & $\beta^{2} \omega_{1}^{2}$ & $2 h_{2} \omega_{1}$ & 1 & 0 \\
& $\ddot{x}_{2} / \ddot{x}_{g}$ & 0 & 0 & $\beta^{2} \omega_{1}^{2}$ & $2 h_{2} \omega_{1}$ \\
$\ddot{x}_{g}$ & $x_{1} / \ddot{x}_{g}$ & $-\beta^{2}(1+\mu) \omega_{1}^{2}$ & $-2 h_{2}(1+\mu) \omega_{1}$ & -1 & 0 \\
& $\left(\ddot{x}_{2}+\ddot{x}_{g}\right) / \ddot{x}_{g}$ & $\beta^{2} \omega_{1}^{4}$ & $2 h_{2} \omega_{1}^{3}$ & 0 & 0 \\
\hline
\end{tabular}

TABLE 2. Optimum Parameters assumption that the external excitation be represented by a stationary white noise, which may be a reasonable idealization for the fluctuating component of the wind pressure. If nonstationary and colored noise is used, these optimal parameter values will be different, although they are not necessarily easy to find. This obviously does not imply that the proposed control system is not effective under these conditions. In fact, the quantification of the effectiveness of the proposed system under these more general conditions will be an interesting subject of future research.

\section{NUMERICAL EXAMPLE}

To illustrate the performance of the proposed mega-subcontrol system, the seismic response of an example building equipped with the proposed mega-subcontrol system as illustrated in Fig. 7(b) was numerically simulated and compared with that of a conventional counterpart without the control system as shown in Fig. 7(a). The two buildings have the same amount of total mass to represent a 200 -m-tall building. The conventional building and the megastructure in the controlled building have the same natural frequencies (values are given in Fig. 7) and the same damping characteristics, which result in a $2 \%$ damping ratio for all vibration modes. This damping ratio $2 \%$ is a typical value for tall steel buildings.

In the mega-subcontrolled building, the mass ratio $\mu$ is 1.0 for all the modes. The stiffness and damping ratio of the substructure are designed to be the optimal values, which minimize the displacement of the megastructure under seismic excitations. According to Table $2, \beta$ should be 0.15 and $h_{2}$ should be 0.35 .

In Fig. 8(a), the transfer function between the displacement of the top mass in the conventional building and the ground acceleration is shown in the solid line. This should be compared with the transfer function between the displacement of the top megamass in the mega subbuilding and the ground acceleration shown in the dashed line. Dramatic reduction at the frequency response of the megadisplacement is achieved by the mega-subcontrol system. A similar effect of the mega subsystem on controlling the acceleration of the substructure is also demonstrated by comparing the transfer function, as shown in Fig. 8(b).

The seismic response of the two building structures was numerically simulated. Table 3 lists the peak values of the mega-subbuilding responses including acceleration at the top submass, relative displacement at the top megamass, base shear coefficient, and the stroke between the top mega and

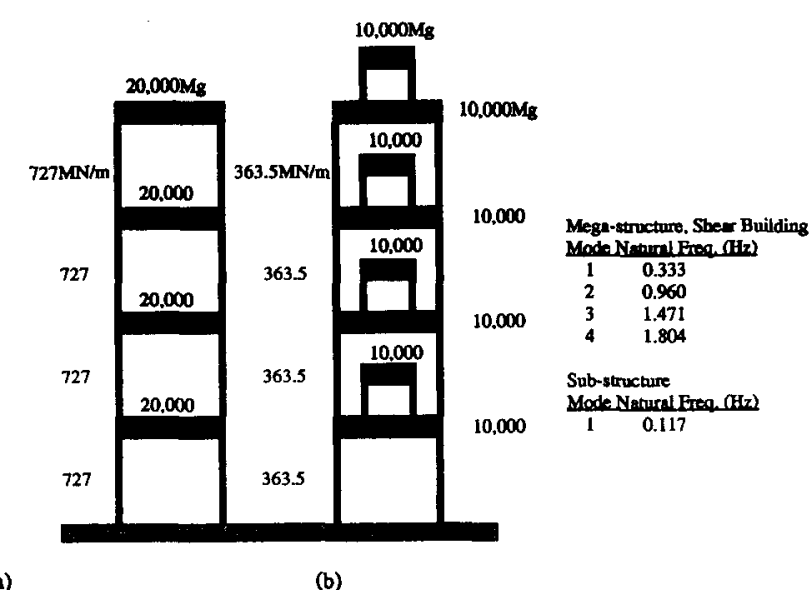

FIG. 7(a). Building without Control; (b) Building with Mega Subcontrol 

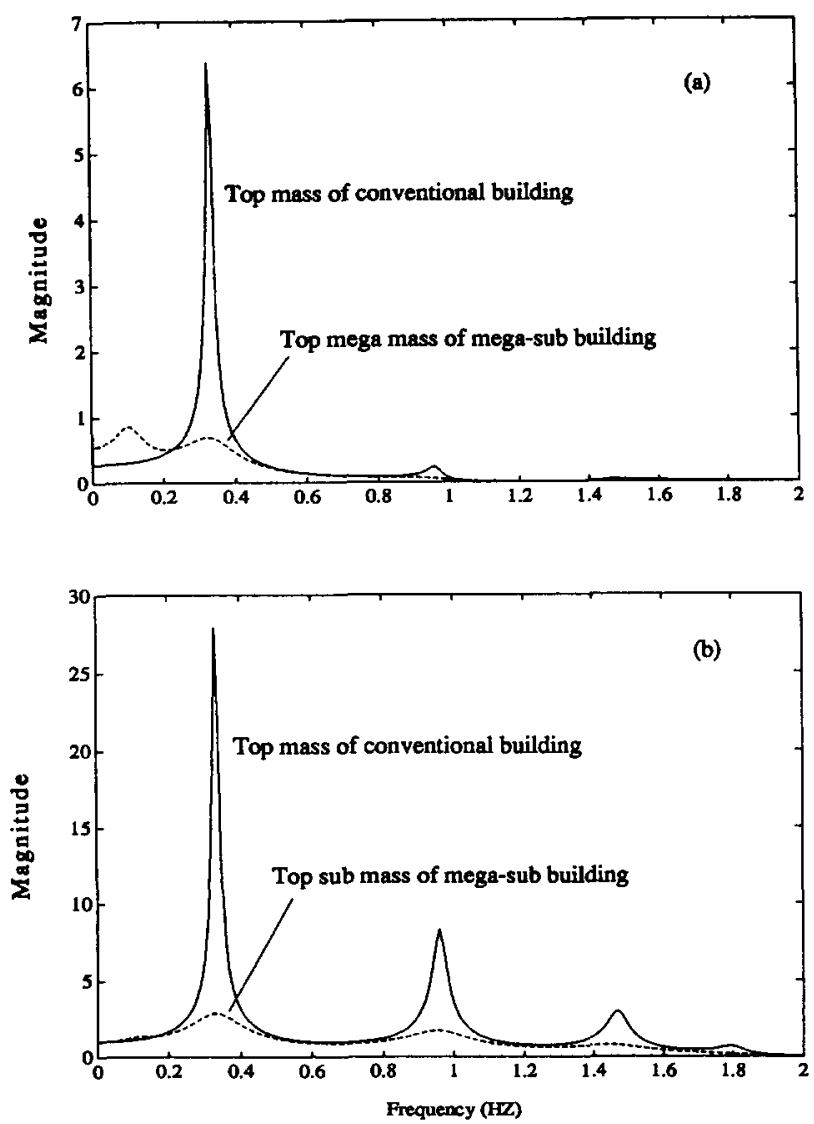

FIG. 8. Comparison of Transfer Functions: (a) Displacement Transfer Function; (b) Acceleration Transfer Function

TABLE 3. Peak Response Subject to El Centro 1940 NS

\begin{tabular}{l|c|c|c|c}
\hline $\begin{array}{c}\text { Mode of control } \\
(1)\end{array}$ & $\begin{array}{c}\text { Top sub- } \\
\text { acceleration } \\
\left(\mathrm{cm} / \mathrm{s}^{2}\right) \\
(2)\end{array}$ & $\begin{array}{c}\text { Top mega- } \\
\text { displacement } \\
(\mathrm{cm})\end{array}$ & $\begin{array}{c}\text { Base shear } \\
\text { coefficient } \\
(\%)\end{array}$ & $\begin{array}{c}\text { Top } \\
\text { stroke } \\
(\mathrm{cm})\end{array}$ \\
\hline Without control & 275.9 & 35.8 & 11.3 & - \\
With control & 20.1 & 15.6 & 4.8 & 19.5 \\
\hline \hline
\end{tabular}

top submasses under the input acceleration, which is the El Centro (1940 NS) earthquake with the normalized peak velocity of $25 \mathrm{~cm} / \mathrm{s}$. In the same table, the corresponding peak response values in the conventional building are also listed. Fig. 9(a) provides the comparison of the time histories of the relative displacements at the top mass of the conventional building (in solid line) and at the top megamass of the mega subbuilding (in dashed line). The response acceleration time histories at the top mass in the conventional building and the top submass in the mega subbuilding are shown in Fig. 9(b).

Table 3 and Fig. 9 clearly demonstrate the significant effectiveness of the mega-subcontrol system in reducing not only the response acceleration to enhance human comfort, but also the base shear coefficient, which is a key design parameter for structural safety. The stroke between the mega and the substructures at the top is $19.5 \mathrm{~cm}$, which is a reasonable value from the viewpoint of structural design.

Although the numerically simulated wind responses are not given in this paper, the performance of the mega-subcontrol system is expected to be similar or even better than that under seismic loads, since wind loads contain less nonstationary components compared with earthquakes.
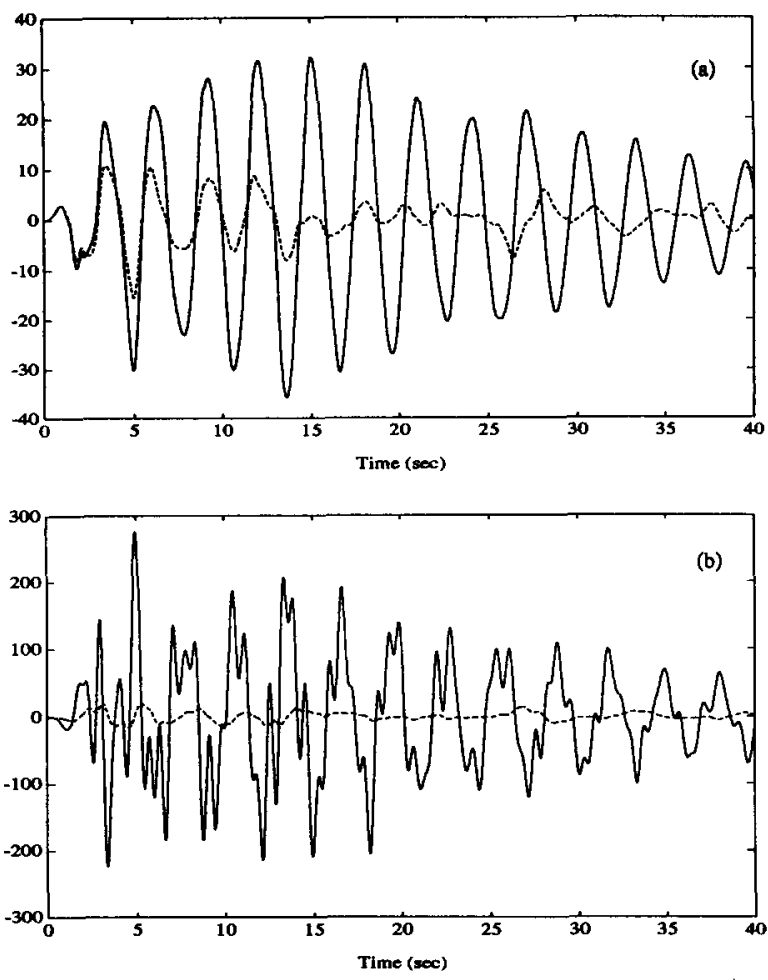

FIG. 9. Comparison of Time Histories: (a) Displacement at Top Megamass (cm); (b) Acceleration at Top Submass (gal)

\section{CONCLUDING REMARKS}

An innovative vibration-control system, which takes advantage of the mega-substructure configuration, was proposed for tall and super tall buildings. This mega-subcontrol system was designed in such a way that the vibration energy (kinetic energy) of the megastructure due to wind or earthquake loads can be transferred into the substructures and then dissipated in the substructures by conventional damping devices. This control system does not require any additional mass as seen in the conventional mass damper systems. The difficulties in the vibration control of tall buildings associated with their high rigidity and slenderness can be overcome by the proposed mega-subcontrol system.

The optimum values of parameters such as the frequency and damping ratio of the substructure were derived for a simplified model of a mega substructure. The dramatic effectiveness of the mega subsystem in controlling building vibration responses to improve human comfort and structural safety was demonstrated through analytical and numerical studies. The simplicity and effectiveness make this unique control system extremely attractive for its future implementation in tall and super tall buildings.

\section{ACKNOWLEDGMENT}

The first writer wishes to acknowledge the support of the National Science Foundation, Arlington, Virginia, under Contract CBS-9223234.

\section{APPENDIX. REFERENCES}

Crandall, H. H., and Mark, W. D. (1963). Random vibration in mechanical systems. Academic Press, Inc., San Diego, Calif.

Kaneko, M., and Mita, A. (1993). "A study on energy flow in a hybrid mass damper and its control algorithms." J. Struct. Constr. Engrg., Japan, No. 449, 103-110.

Mita, A., and Feng, M. Q. (1994). "Response control strategy for tall 
Buildings using interaction between mega- and sub-structures." Proc., Ist Int. Workshop on Civ. Infrastructure Systems, Nat. Taiwan Univ., Taipei, Taiwan, 329-341.

Mita, A., and Kaneko, M. (1992). "Hybrid versus tuned or active mass dampers for response control of tall buildings." Proc., Ist Int. Conf. on Motion and Vibration Control, Japan Soc. of Mech. Engrs., Tokyo, Japan.

Warburton, G. B. (1982). "Optimum absorber parameters for various combination of response and excitation parameters." Earthquake Engrg. and Struct. Dynamics, 10(3), 381-401. 